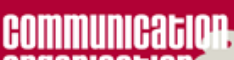
Orgallostion.
Communication et organisation

Revue scientifique francophone en Communication

organisationnelle

$50 \mid 2016$

Engagement entrepreneurial et territoires

\title{
Rénovations urbaines mises en récit pour de nouvelles représentations des cités
}

Urban renovations put into stories for new representations of estates

\section{Natacha Cyrulnik}

\section{OpenEdition}

Journals

Édition électronique

URL : http://journals.openedition.org/communicationorganisation/5382

DOI : 10.4000/communicationorganisation.5382

ISBN : 979-10-300-0127-3

ISSN : 1775-3546

Éditeur

Presses universitaires de Bordeaux

Édition imprimée

Date de publication : 1 décembre 2016

Pagination : 111-122

ISBN : 979-10-300-0069-6

ISSN : 1168-5549

Référence électronique

Natacha Cyrulnik, "Rénovations urbaines mises en récit pour de nouvelles représentations des cités », Communication et organisation [En ligne], 50 | 2016, mis en ligne le 01 décembre 2019, consulté le 16 février 2021. URL : http://journals.openedition.org/communicationorganisation/5382 ; DOI : https:// doi.org/10.4000/communicationorganisation.5382 


\title{
Rénovations urbaines mises en récit pour de nouvelles représentations des cités
}

\author{
Natacha Cưrulnik ${ }^{1}$
}

Même si les directives de la politique de la ville ont été revues par le gouvernement en établissant une nouvelle carte des territoires dits prioritaires selon une moyenne des sources de revenus, de nombreuses cités ont bénéficié ces dernières années de Plans de Rénovation Urbains. Nicolas Sarkozy a finalement peu vanté dans son bilan présidentiel le fait que de très gros investissements avaient été menés dans les cités en France. Peut-être parce que les termes de «racailles » et de «karchers » ont participé, par ailleurs, à une plus grande stigmatisation de ces quartiers! Entre investissements en termes de moyens et d'équipements, abus de langage et représentations sociales en général, les cités et leurs images sont en mutation.

À partir d'un travail audiovisuel entrepris depuis 1999 dans les cités du sud de la France (dans le cadre notamment de la série documentaire «habiter le territoire» qui regroupe la cité Berthe de La Seyne-sur-mer, le quartier Le Carami à Brignoles, l'ensemble des quartiers de La Ciotat, et la cité Air Bel à Marseille) un tableau se dresse sur la vie au quotidien dans les cités et sur ses transformations. Cette série documentaire offre aux habitants l'occasion de prendre la parole au sujet de leur vie quotidienne, et de formuler leurs désirs de changement. Elle permet également au spectateur qui va voir le(s) film(s) de mieux comprendre la situation, et d'en acquérir une connaissance plus sensible à travers cette expérience filmique (Niney 2000 et 2002). Elle offre aussi aux architectes, aux élus, aux responsables des collectivités territoriales, aux entrepreneurs et autres bailleurs, l'occasion d'entendre ces paroles et la réflexion qui se développe à partir de ce qui émerge du terrain, afin qu'ils puissent les prendre en compte dans un processus de concertation. Le film documentaire se propose comme un objet, voir un outil, qui favorise l'échange: les habitants peuvent faire entendre leur parole en racontant leur quotidien

1 Natacha Cyrulnik est documentariste et maitre de conférences en Sciences de l'Information et Communication à l'Université d'Aix-Marseille, au sein du Laboratoire ASTRAM. Elle est la responsable de la spécialité Production et réalisation au département Satis (Sciences Arts Techniques de l'Image et du Son) à Aubagne. Ses travaux portent d'une manière générale sur les représentations des territoires par le biais du film documentaire. 
et en formulant leurs revendications éventuelles: et les décideurs, s'ils ne sont pas déjà présents dans le film projeté, les entendent à travers le film pour y répondre ensuite à l'occasion du débat qui suit la projection. Les propos des habitants comme des décideurs se mêlent, ils se nuancent, et aident ainsi à mieux « vivre-ensemble».

\section{Le documentaire, un dispositif socio-technique et une méthode pour appréhender un territoire}

Le film documentaire se propose ici comme un dispositif socio-technique qui donne la parole et la fait entendre de manière sensible. Nous prenons ici le terme de «dispositif» selon les propos de Giorgio Agamben, «(...) J'appelle dispositif tout ce qui a d'une manière ou une autre, la capacité de capturer, d'orienter, de déterminer, d'intercepter, de modeler, de contrôler et d'assurer les gestes, les conduites, les opinions et les discours des êtres vivants» (2006: 31). C'est la transformation qui s'opère chez l'homme qui importe dans ce dispositif. "J'appelle sujet ce qui résulte de la relation, et pour ainsi dire, du corps à corps entre les vivants et les dispositifs» (Agamben 2006: 32). Ces précisions insistent sur la place de l'humain (du sujet) et de ses évolutions possibles. Le thème du documentaire traite aussi de la transformation urbaine à travers le regard qu'en portent les hommes et ce qu'ils en disent. Les transformations urbaines et humaines sont au cœur du débat.

Dans le cadre de ce dispositif, la caméra place déjà le «filmé» dans une position qui relève d'une approche médiologique (Debray 2000) comme le rappelle Daniel Bougnoux: "sur ces milieux, indissociablement sociaux et techniques, qui façonnent et recyclent nos représentations symboliques, et nous permettent de tenir ensemble» (Bougnoux 1998:67). Elle va donc permettre de travailler sur les représentations sociales et symboliques quévoquent les cités et leurs habitants. Daniel Bougnoux ajoute «loutil numérique encourage[ant] l'individualisme, tant au niveau de l'expression, de l'émission que de la réception des messages; il pose du même coup la question de savoir comment montrer encore le général, le bien commun ou un espace public» (Bougnoux 2007:46). La présence première de la caméra dans les rues d'une cité suscite un évènement qui va déjà favoriser une mise en relation de tous ces interlocuteurs (Lamizet 2006). Elle implique ensuite chez l'habitant qui se retrouve en face un questionnement sur l'espace public: pourquoi une caméra dans les rues de cette cité? Ils vont ainsi parler d'eux, à titre individuel et collectif, et de leur environnement. Le film interroge de fait la place de l'homme au sein de cet espace public. Le rapport entre l'homme et son territoire est posé en tant que questionnement comme en termes de méthode par l'intermédiaire de la caméra et du film documentaire qui en découle. Les deux se développent en cohérence l'un avec l'autre. Le sujet et la forme avancent ensemble. Traiter de la mise en récit d'un territoire doit donc laisser une grande place aux 
représentations symboliques, qui elles-mêmes participent à l'affirmation d'un lien fédérateur pour les habitants de ces territoires ainsi narrés (Cyrulnik et Meyer, 2016).

La caméra en tant qu'objet technique et le film documentaire auquel elle donne naissance (et qu'elle suggère déjà par sa présence) proposent un dispositif qui va favoriser l'échange au moment du tournage, et lors du débat qui suit généralement la projection de ce genre filmique. Les formes situationnelles qu'offre le dispositif du film documentaire (préparation, tournage, montage, projection et débat) deviennent des étapes méthodologiques (Cyrulnik 2008) pour favoriser la prise de parole par la re-formulation phénoménologique (Paillé et Mucchielli 2005), l'articulation d'une pensée, la composition d'un récit, jusqu'à la fédération d'une nouvelle communauté de spectateurs (Cyrulnik 2015).

C'est dans une approche compréhensive (Paillé et Muchielli 2005), dans le cadre d'une observation participante (Winkin 2001) et en se basant sur des codes d'échanges établis (Paillé et Mucchielli 2005) au moment du tournage et qui se prolongent ensuite dans la salle, que l'évolution urbaine et humaine de ces cités est appréhendée. Cette articulation permet de présenter des faits (plans d'urbanisme, projets architecturaux, plans de financement en lien avec cela, mixité privé/public, etc.) avec un certain recul et en prenant en compte des différents discours tenus à ce sujet. Même si ces termes correspondent plutôt au vocabulaire de ceux qui travaillent au projet urbain, et qu'ils sont rarement utilisés par les habitants, ceux-ci parlent tout de même de cela d'un point de vue phénoménologique. Chacun expose son point de vue (à l'écran puis dans la salle), avec toute la volonté d'objectivation de chacun et toute la subjectivité qui débordent à travers ces re-formulations phénoménologiques.

\section{Formes de discours et niveaux de représentations}

\section{Formes de discours}

Les films traitent des discours qui sont tenus au sujet du territoire. Un tel dispositif offre aux habitants, et aux personnes qui travaillent sur le projet urbain (architectes, urbanistes, paysagistes, élus, responsables du Gip ou de l'Anru, etc.) l'occasion d'échanger ensemble à ce sujet. Il ne s'agit en aucun cas de les opposer mais bien au contraire de lier leurs propos afin qu'un projet urbain puisse se construire dans un véritable processus de concertation, ensemble ${ }^{2}$. Les propos émergent face à la caméra, ils se mêlent dans le film et se développent les uns avec les autres au fil du montage, et ils se nuancent encore dans la salle lors du débat. Ces étapes méthodologiques participent à la construction d'un discours, mais surtout suscitent une réflexion globale qui

2 Même si la plupart des habitants des cités de Marseille ne veulent plus entendre ce terme de «concertation», tellement il leur a été «rabâché» pour ne rien voir se réaliser par la suite, et tellement ils considèrent qu'il est usurpé maintenant! (sic) 
va être poursuivie dans la salle. En définissant une méthode pour aborder ces territoires et ses habitants, nous leur proposons de composer un récit au sujet de cet environnement, dans un registre qui fluctue entre l'intime et le collectif. Cette affirmation vis-à-vis des uns et des autres ${ }^{3}$ permet de passer d'un récit d'ordre général à un discours incarné par des personnes, qui devient plus vivant, plus subjectif, donc à modérer par la suite. Gérard Genette (1966: 161) souligne que ces deux termes de «récit» et de «discours» sont théoriquement opposés: le discours se caractérise par le fait d'être rattaché à un émetteur, tandis que dans le récit, personne ne parle, si bien que le lecteur (spectateur) ne se demande pas qui parle pour recevoir la signification (Le Corf 2016). Les témoignages sont incarnés, vivants, vécus dans un film documentaire. Ils deviennent donc des discours, avec l'articulation entre le fond de ce qui est dit au niveau du vécu dans ces cités, mais aussi en prenant en compte la forme plus ou moins revendicative des propos. Le fond et la forme se mêlent.

\section{Représentation filmique}

Ainsi, dans «Ceux qui pensent le projet urbain, ceux qui le vivent » (2011), par exemple, les propos de lélu à la Ville alternent avec ceux de différents habitants sur quelques sujets concernant ce qui était encore au stade de projet de rénovation urbaine à l'époque. La clarté de ses propos, sa volonté de bien présenter les différents éléments à prendre en compte, sa tenue vestimentaire, sa situation derrière un bureau contrastaient avec la spontanéité des habitants en situation dans les rues de la cité Berthe de La Seyne-sur-mer. Ceux-ci semblaient ainsi plus vivants, et avaient alors tendance à susciter plus d'empathie (Le Marquis 2015). Il devenait alors important de proposer à cet élu ${ }^{4}$ d'aller sur le terrain à son tour pour ancrer ses propos dans une réalité alors que son discours pouvait apparaitre comme étant trop politique, trop «propre» et trop formaté dans la première situation. Les différents niveaux de discours évoluaient clairement selon les situations. L'ancrage sur le terrain s'est avéré essentiel pour faire entendre les propos de tous à "armes égales».

La représentation filmique se met en place selon l'articulation des propos, leur évolution. Le film se modèle au fur et à mesure de la forme des discours, d'un point de vue à la fois esthétique et rhétorique (Soulez 2011). Le documentaire se construit: il donne une trace de cette réalité, il la représente. Ce «re» de «représentation» (Bougnoux 2006) permet une distanciation, sort ainsi de la sidération, et aide à mieux comprendre la situation exposée. Représenter un tel territoire, c'est choisir la manière de le faire comprendre au mieux. C'est en tout cas ce que peut offrir un film documentaire.

3 Même quand la personne est seule face à la caméra, cet objet caméra évoque les nombreux spectateurs à venir 4 Le montage se pense aussi au moment du tournage, puisqu'il s'agit d'articuler une pensée sur l'évolution urbaine et humaine durant ces films. 
Le documentaire propose aussi des moments de projection au sein des quartiers afin d'échanger avec le(a) réalisateur(trice) à l'issue de la projection. Cette sorte d'événement (Lamizet 2006) va susciter le débat avec la salle, favorisant ainsi l'échange entre tous les spectateurs, de l'habitant de la rue d'à côté au maire, en passant par l'architecte, le responsable du GIP ou encore par l'élu à la politique de la ville. Un nouveau récit se compose alors entre tous ces spectateurs, qui s'appuie sur les propos qui viennent d'être projetés sur le grand écran pour les nuancer à leur tour dans la salle. C'est un nouveau processus de concertation qui se met en place par cette mise en récit basée principalement sur les représentations de chacun: représentation filmique, représentation de la cité, représentation des élus, représentation sociale, représentation de soi, etc... et un nouvel engagement entrepreneurial se mettra en place sur la base de ces représentations.

\section{Représentation sociale}

Cette représentation filmique se construit au présent lors du tournage et au moment du débat dans la salle, permettant ainsi de nuancer les représentations d'ordre social cette fois qui caricaturent souvent ces cités comme des zones dangereuses, notamment dans les médias (Avenel 2005: Moscovici 1984; Maigret et Macé 2005). Définir avec les habitants de nouveaux repères ou de nouveaux codes pour mieux les comprendre permet au spectateur à son tour de mieux percevoir cette réalité de manière sensible. Les représentations sociales évoluent: elles se nuancent à partir de l'expérience vécue lors de la projection et des échanges qui suivent. Cette dimension sociale est importante à traiter, et le fait qu'une parole soit portée par un individu à l'écran favorise cela. La personne ainsi incarnée est perçue comme étant issue de la réalité puisque le spectateur sait qu'il s'agit d'un documentaire. Il intègre ces images comme étant issues d'un contrat tacite entre lui et le réalisateur qui s'engage d'un point de vue éthique à ce que ces images soient issues de la réalité (Lioult 2004). Il y a donc une part de vérité, même si tous les propos tenus à l'écran témoignent aussi d'une véritable dimension subjective ${ }^{5}$ ! Cette forme d'implication, d'engagement, va participer à un processus de transformation qui devrait mener à une forme de responsabilisation pour l'ensemble des participants (filmés et spectateurs) (Boal 1977).

Ces documentaires vont favoriser l'acquisition d'une meilleure connaissance de ces territoires particuliers en les comprenant mieux à travers les témoignages offerts (Niney 2000 et 2002). Ainsi, les habitants de chacune des villes vont pouvoir proposer par le film, l'expérience d'une appréhension différente de leur cité. Les représentations sociales évoluent. Et les entreprises vont y participer en investissant dans ces cités pour valoriser leur engagement social

5 C'est aussi cela qui fait la force du témoignage! 
et leur image de marque, tout en favorisant la mixité public/privé, comme nous le verrons plus loin.

\section{Représentation de soi}

La transformation possible dans ce dispositif peut se faire aussi par rapport à la représentation de soi, pour les «filmés» comme pour les spectateurs. Ceuxci se mettent en situation d'exposition face aux autres ${ }^{6}$. La caméra catalyse des comportements pour ce public potentiel qu'elle évoque, incitant à se tenir un peu plus droit, à articuler un peu plus, ou même à se cacher derrière une cagoule quand les jeunes croient qu'elle est là pour un «reportage pour TF1 ${ }^{7}$, avant de l'enlever finalement si on prend le temps, et d'essayer de formuler une idée au mieux pour se montrer le plus intéressant possible pour les futurs spectateurs. En plus de chercher à changer les représentations sociales souvent caricaturales dont elle souffre, la personne filmée compose aussi une autre représentation de soi. Le processus de transformation se fait donc au niveau urbain, social et intime.

Avec une représentation filmique sous la forme d'un documentaire, des représentations sociales qui se nuancent au fur et à mesure que l'on expérimente le dispositif, et des représentations de soi qui permettent l'affirmation d'un discours dans l'espace public, comment le processus de concertation mis en place peut-il à la fois favoriser l'expression des désirs des habitants, la volonté des élus, les parti-pris des architectes et autres urbanistes, et l'implantation des entreprises dans une dimension plus économique?

\section{Engagement entrepreneurial et participation citoyenne par ces représentations}

Les films documentaires inscrits dans cette série proposent de favoriser la mise en récit de ces territoires avec leurs revendications, différences, similitudes, nuances, spécificités, etc. Ils permettent d'expérimenter autrement ces lieux tout en en affirmant leur complexité. À travers les récits filmiques qui se composent, une nouvelle manière d'interpréter ces territoires est proposée.

Nous sommes en train de décrire un dispositif socio-technique qui devient de plus en plus participatif, pour les habitants et les spectateurs, comme pour les décideurs. L'engagement des collectivités favorise ces mutations urbaines. Des entrepreneurs ${ }^{8} s^{\prime} y$ investissent aussi de plus en plus afin de favoriser une mixité

\footnotetext{
6 Ou face à la caméra qui suggère déjà les futurs spectateurs qui vont venir après... ou lors du tournage quand des habitants, s'ils sont derrière la caméra pour entendre les propos tenus par d'autres lors du tournage, peuvent s'approcher de la caméra pour les nuancer à leur tour!

7 S'enfermant dans ces clichés parce qu'ils croient que les caméras sont là pour ça, et s'enfermant ainsi dans les représentations sociales dont ils souffrent ! Au bout de quelques minutes, voire quelques mois (le temps est particulièrement important à prendre en compte dans ces situations-là), d'autres relations se tissent et une autre forme de témoignage peut émerger et se développer.

8 Les entrepreneurs, et même les bailleurs, en viennent également à financer un peu les films de cette série
} 
sociale. La transcription des intentions urbaines et architecturales, sociales et économiques définit les orientations qui font sens pour ces transformations. Ainsi le principe de "résidentialisation", qui consiste à regrouper deux ou trois bâtiments et à délimiter «en dur» des résidences, est le parti-pris revendiqué par la ville de La Seyne-sur-mer: alors que c'est l'ouverture de toutes les frontières virtuelles en termes d'aménagement du territoire qui s'est mise en place entre les quartiers Abeille-Maurelle-Matagot à La Ciotat, par exemple. La mise en forme de ces cités évolue selon les discours et les partis-pris qui leur sont associés. Le récit que les différentes collectivités font le choix de composer, impacte clairement sur la future manière de vivre au quotidien dans ces quartiers.

Aussi les entrepreneurs, et même les bailleurs, construisent-ils des logements privatifs dans ces zones sensibles dans une logique de mixité sociale. Ils affichent ainsi une nouvelle image de ces cités. S'ils le font c'est bien parce que des directives gouvernementales pour favoriser les logements sociaux dans les villes sont mises en place: et c'est aussi l'occasion pour les « très grosses» entreprises du BTP de changer leur propre image en s'engageant dans ces quartiers stigmatisés. Si la volonté économique est plutôt en lien avec cette volonté de changer cette image de marque, elle s'avère équilibrée d'un point de vue financier également. Malheureusement, les habitants expliquent que les appels d'offres sélectionnent en priorité le moins cher et déplorent, par exemple, que «les stores mis en place s'envolent au premier coup de mistral!». L'image de marque de la cité n'est donc pas valorisée: le processus entrepreneurial initié n'est pas abouti. Ces réalités ancrées dans le quotidien enferment toujours les habitants dans les représentations sociales dont ils souffraient déjà auparavant.

Mais l'engagement entrepreneurial existe aussi dans les obligations que les entreprises du BTP ont souvent à travers les contrats signés avec les villes: elles sont tenues d'embaucher des jeunes de ces quartiers dits «défavorisés» pour les travaux de rénovations. Même si les jeunes n'ont souvent pas les diplômes suffisants pour conduire des "gros engins» pour les travaux (engins qui par ailleurs, les font rêver), il y a toujours un faible pourcentage qui est embauché. Alors, ces jeunes travaillent chez eux, pour eux, expliquent à leurs voisins ce qui va être fait, se font la plupart du temps une première expérience professionnelle à mettre dans leur $\mathrm{CV}$, etc. Cette obligation qui correspond souvent à des contrats aidés (donc moins onéreux pour les entreprises) apporte beaucoup pour la paix et le bien-être dans la cité. Ces contrats s'inscrivent dans une forme de concertation puisque ces jeunes expliquent les travaux à leurs voisins, mais aussi affichent une forme de fierté et d'engagement citoyen

documentaire afin de participer à ces échanges citoyens, et de mieux s'inscrire dans ce paysage urbain et social, comme c'est le cas à Marseille. 
qui leur permet de se mettre en avant, et qui valorise aussi ces travaux qui vont ainsi être plus respectés ${ }^{9}$ par la même occasion!

Toutes ces situations en lien avec les politiques, aménageurs, bailleurs et autres architectes sont précisément les sujets abordés dans les films. Les films offrent tous ces témoignages. Par l'expérience faite à travers ces films, un savoir s'acquiert. Dans cette même dynamique, ces situations participent à une meilleure cohésion sociale. Par la prise de parole de ces jeunes travailleurs filmés, une forme de solidarité et d'empathie est affichée. À partir de l'engagement entrepreneurial, c'est l'engagement citoyen qui est maintenant favorisé.

À travers ce que les habitants choisissent de dire de leur cité, et leur manière de le formuler, un portrait territorial se dessine. Cette nouvelle proposition en termes de concertation semble aller dans le sens proposé par les nouvelles directives gouvernementales dans la mesure où les conseils de citoyens sont en train de se mettre en place à l'heure où nous écrivons ce texte. La volonté est de stimuler l'engagement citoyen. La participation est également le fondement de notre approche audiovisuelle. Elle l'est aussi en termes d' "empowerment», comme le revendique Marie-Hélène Bacqué (Bacqué et Biewener 2013) qui avait d'ailleurs initié les processus pour la définition de cette nouvelle politique de la ville avec le secrétaire d'état François Lamy quand il a été nommé au début du mandat de François Hollande. Cette logique participative revendiquée pour faire évoluer les cités par ces décisionnaires, entre également en jeu pour construire ces représentations filmiques. Le principe de concertation est ainsi revisité, avec la dimension historique en plus qui permet une nouvelle lecture de ces mutations urbaines puisque ce travail audiovisuel a débuté en 1999.

La dimension artistique importe aussi dans ce processus puisquelle favorise l'expression individuelle et collective à travers l'expérience offerte (Dewey 1915). L'Art apporte un imaginaire et une symbolique qui aident à la prise de parole au sujet de ces territoires sensibles, et favorisent des mises en récit plus ou moins fictionnelles qui révèlent une réalité vécue au quotidien. C'est en cela que le film documentaire qui se construit avec les habitants devient un outil participatif et porteur de nombreuses projections possibles pour rêver d'un monde meilleur ensemble. L'Art propose un processus de transformation qui mène à une implication plus forte de la personne. Il favorise l'engagement citoyen sur le même principe qu'Augusto Boal (1977) l'avait décrit dans sa méthode du théâtre forum. Son but consistait à faire évoluer un spectateur qui pourrait paraître passif, en véritable acteur au sein de sa cité. Ce «spect'acteur» devient de plus en plus impliqué dans sa cité de la même manière que notre acteur (ou celui qui est devant la caméra) s'expose pour se faire entendre par de futurs spectateurs qui prendront sa parole en compte lors des échanges après la projection, pour la reformuler à leur tour. Ce qui nous importe, c'est

9 C'est-à-dire qu'ils ne seront pas vandalisés. 
que tous (acteurs, spectateurs, habitants, décideurs et autres), interagissent pour formuler ensemble les évolutions urbaines à mettre en place pour une meilleure vie au quotidien dans ces quartiers. Le caractère utopiste de cette intention se révèle au final dans la prise de «petites» décisions, souvent très simples, qui ont l'avantage de mettre tout le monde d'accord: des habitants revendiquent le droit de mettre leur linge à sécher à leur fenêtre et les décideurs acceptent: le paysagiste propose différentes formes de grilles que les habitants peuvent choisir: les habitants demandent à ce qu'un passage clouté soit dessiné dans l'alignement du portail de leur résidence, par exemple. S'il est la plupart du temps difficile de demander aux habitants d'avoir une vision urbaine globale de leur quartier, le simple fait pour eux de pouvoir impacter sur ces petites décisions, et se faire entendre, offre une forme de (ré-) conciliation qui propose une forme de "mieux-vivre-ensemble».

Les notions d' «empowerment» et de «spect'acteur» associées à l'apport de la dimension artistique, valorisent chacune à leur manière l'engagement citoyen. Les films documentaires favorisent cela. Il s'agit de placer ces habitants dans une situation où ils se sentiraient appartenir à une grande cité, celle qui correspond au sens politique du terme chez les grecs. Les habitants sont au cœur du dispositif au même titre que les décideurs et vice-versa : tous ensemble, que ce soit à l'écran ou dans la salle, à essayer d'exposer des situations et de trouver des solutions. Une logique d'échange, de participation et d'engagement entre dans une dimension démocratique. Aborder un territoire tel que celui de(s) (la) cité(s) interroge ses représentations possibles d'un point de vue politique. C'est la place de l'homme au sein de sa cité qui est interrogée. La dimension politique est aussi au cœur de ce dispositif de concertation.

\section{BIBLIOGRAPHIE}

AGAMBEN Giorgio (2006), Quiest-ce quiun dispositif? Paris, Rivages Poches, Petite bibliothèque

AVENEL Cyprien (2005), Sociologie des «quartiers sensibles», A.Colin.

BACQUE Marie Hélène et BIEWENER Carole (2013), L'empowerment, une pratique émancipatrice, Ed. La Découverte, coll. Politique et sociétés.

BOAL Augusto (1977), Théâtre de l'opprimé, Paris, Ed. La Découverte Poches, 1996.

BOUGNOUX Daniel (1998), Introduction aux sciences de la communication, Ed. la Découverte, 2001.

BOUGNOUX Daniel (2006), La crise de la représentation, Paris, Ed. La découverte.

BOUGNOUX Daniel (2007), «La communication publique entre représentation et participation", p.41-50. In Actes du colloque La communication publique, approche, évolutions et enjeux. Tunis: IPSI, Fondation Konrad Adenauer.

CYRULNIK Natacha (2008), Représenter le monde et agir avec lui, la méthode du documentaire de création, Thèse de doctorat, sous la dir. de P. Dumas et F. Renucci, Université du Sud - Toulon Var. 
CYRULNIK Natacha (2015), «Le documentaire, un espace de liberté pour une nouvelle communauté», in Spicilège dirigé par Gino Gramaccia, Revue Française des Sciences de l'Information et de la Communication n ${ }^{\circ}$ 7, octobre 2015.

CYRULNIK Natacha et MEYER Vincent (2016), Des territoires et des récits: milieux, médias et médiations, in La mise en récit des territoires méditerranéens: langages et objets, dirigé par Natacha Cyrulnik et Vincent Meyer, revue du Gerflint - Synergies mondes méditerranées n ${ }^{\circ}$ 5/2015, mars 2016, p.7-18

DEBRAY Régis (2000), Introduction à la médiologie. PUF, Collection Premier Cycle.

DEWEY John (1915), L'art comme expérience, Paris, Coll. Folio Essais, Gallimard, 2005.

GENETTE Gérard (1966), Frontières du récit, in Communications, n 8, p.152-163.

LAMIZET Bernard (2006), Sémiotique de l'évènement, Paris, Lavoisier.

LE CORF Jean Baptiste (2016), Le récit métropolitain, composante de la communication touristique. Les cas de Marseille et d'Istanbul face au label «capitale européenne de la culture", in La mise en récit des territoires méditerranéens: langages et objets, dirigé par Natacha Cyrulnik et Vincent Meyer, revue du Gerflint - Synergies mondes méditerranées n 5/2015, mars 2016, p.145-161.

LEMARQUIS Pierre (2015), L'empathie esthétique, entre Mozart et Michel-Ange, Odile Jacob, Paris.

LIOULT Jean-Luc (2004), À l'enseigne du réel, penser le documentaire, Marseille, Publications de l'Université de Provence.

MOSCOVICI Serge (1984), sous la dir. de, Psychologie sociale, coll. Quadrige manuels, PUF.

MAIGRET E. et MACE E. (2005), Penser les médiacultures - Nouvelles pratiques et nouvelles approches de la représentation du monde, Paris, A. Colin/INA.

NINEY François (2000), L'épreuve du réel à l'écran. Essai sur le principe de réalité documentaire, Deuxième édition, De Boeck Université, Bruxelles.

NINEY François (2002), La poétique documentaire comme forme de connaissance, États généraux du film documentaire, (consulté le 20.07.07), disponible sur:

http://www.lussasdoc.com/etatsgeneraux/2002/sem_poetique.php4

PAILLE Pierre et MUCCHIELLI Alex (2005), L'analyse qualitative en sciences humaines et sociales, Paris, A. Colin.

SOULEZ Guillaume (2011), Quand le film nous parle, Rhétorique, cinéma, télévision, Coll. Lignes d'art PUF

WINKIN Yves (2001), Anthropologie de la communication - de la théorie au terrain, Paris, Ed. Le Seuil.

\section{Filmographie}

- "Ceux qui pensent le projet urbain, ceux qui le vivent», 2011, 80', documentaire de Natacha Cyrulnik dans la série «Habiter le territoire», prod. La Compagnie des Embruns. 
Résumé: La série de films documentaires «Habiter le territoire» questionne les plans de rénovations urbains mis en place dans de nombreuses cités en France. Elle propose un processus de concertation par le biais du documentaire qui favorise l'échange entre les habitants et les décideurs de ces aménagements territoriaux. Les différentes formes de discours qui se développent dans le film ou lors des débats à l'issue de la projection permettent de reformuler les situations pour construire de nouvelles représentations filmiques, sociales, individuelles et collectives. Celles-ci favorisent l'engagement entrepreneurial et la participation citoyenne.

Mots-clés : représentations, discours, territoire, concertation et implication.

Abstracts : The series of documentary movies " to Live in the territory" questions the urban plans of renovations set up in many «cités» in France. She proposes a process of dialogue by the documentary which favors the exchange between the inhabitants and the decision-makers of these territorial developments. The various forms of speech which develop in the movie or during the debates at the end of the projection allow to reword the situations to build it of new cinematic, social, individual and collective representations. These favor the entrepreunarial commitment and the participation citizen.

Keywords : representations, speech, territory, dialogue and implication. 
